Contributions to Game Theory and Management, XIII, 402-414

\title{
On Control Reconstructions to Management Problems
}

\author{
Nina N. Subbotina ${ }^{1,2}$ \\ 1 Krasovskii Institute of Mathematics and Mechanics UrB RAS, \\ 16 S.Kovalevskaya Str., Yekaterinburg, 620108 Russia \\ E-mail: subb@uran.ru \\ 2 Ural Federal University, \\ 19 Mira Str, Yekaterinburg, 620002 Russia
}

\begin{abstract}
We present and discuss a new approach to solutions of control reconstruction problems in real time. The suggested solution based on necessary optimality conditions for auxiliary calculus of variation problems with concave-convex discrepancy functional.
\end{abstract}

Keywords: optimal control, nonlinear systems, control reconstruction, calculus of variation, concave-convex discrepancy.

\section{Introduction}

Dynamic controlled processes are widely found in surrounding life: in engineering, business, economics, medicine, social changes, traffic, education, etc. See, for example, (Bellman, 1957; 8; Leitmann, 1962; Krasovskii, 1968; Michel, 1977; Petrosjan and Zen kevich, 1996). Importance of management for these processes lead to the need to study and solve not only direct control problems aimed at constructing controls that optimize quality criteria. For predicting, planning and decision making in the future, it is important also to analyse and solve inverse problems directed on control reconstruction and based on inaccurate measurements of realized motions. See, (Sabatier, 2000; Osipov et al., 2011; D'Autilia et al., 2017; Liu et al., 2018), where many methods were suggested to solve different inverse problems. The methods applied ideas and results of algebra, geometry, functional analysis, the theory of approximations, the theory of perturbations and so on. But reconstruction problems are still actual and many new applied inverse problems require new effective methods.

It should be underlined the approach suggested by Yu.S. Osipov and A.V. Kryazhimskii (Kryazhimskii and Osipov, 1984, Osipov and Kryazhimskii, 1995). It sources in the theory of optimal feedbacks (Krasovskii and Subbotin, 1988) developed by N.N. Krasovskii's school. The approach is more close to the presented below one. It uses the method of extreme aiming to motions of a guide model, whose dynamics copy dynamics of the original controlled system. So, this approach to solution of inverse problems appeals a coupled dynamic system: the original dynamic system and a similar guide system.

The presenting method was suggested in (Subbotina and Tokmantsev, 2013; Subbotina et al., 2015). There was used a new variational approach based on solutions of auxiliary problems of calculus of variation with integral regularized discrepancy functionals. According to necessary optimality conditions in these variational problems, a coupled system is also introduced: the original system and an additional system of conjugate variables. The key new feature of the approach is that

\footnotetext{
* This work was supported by the Russian Foundation for Basic Research under grants No.20-01-00362 and No. 18-01-00221
} 
the discrepancy is concave-convex. This character of discrepancy provides oscillations motions of the reconstructed system around inaccurate measurements of the real states of controlled system.

At first papers this approach was developed for solving inverse problems at the end of the controlled process by the use a posteriori information about history of measurements of states (Subbotina and Krupennikov, 2017; Krupennikov, 2018; Subbotina and Krupennikov, 2018). Now the same ideas are developed to solve dynamic reconstruction problems in real time.

Note, that both mentioned above approaches can be also considered as developments of Tikhonov regularization method (Tikhonov, 1963).

The paper is organized as follows. The section Statement contains description of a control reconstruction problem (CRP): controlled dynamics, incorrect measurements and main hypotheses on input data, and also a definition of solution of CRP. The next section Auxiliary Constructions contains statement of auxiliary problems of calculus of variations (CVP) and necessary optimality conditions in CVP. An algorithm of control reconstruction by the use CVP is described and discussed in the section Solutions CRP. Illustrative examples of numerical solutions of control reconstruction problems in macroeconomics and navigation are exposed at the section Examples.

\section{Statement}

We consider control systems with dynamics of the form

$$
\dot{x}(t)=G(x(t), t) u(t), \quad t \in[0, T]
$$

where $x(t) \in R^{n}$ are state variables. The admissible controls $u(\cdot):[0, T] \rightarrow R^{m}, m \leq$ $n$, are piecewise continuous functions with finite number of points of discontinuity, and they satisfy the following geometric restrictions.

$$
u(t) \in U, \quad t \in[0, T]
$$

where $U=\left\{a_{i} \leq u_{i} \leq b_{i}, i=1, \ldots, m\right\} \subset R^{m}$.

It is assumed that a trajectory $x^{*}(\cdot):[0, T] \rightarrow R^{n}$ of system (1) is realizing in real time. Discrete measurements $x\left(t_{k}, \delta\right)$ of this basic trajectory $x^{*}(\cdot)$ become to be known in real time at instants $t_{k}$.

Measurements $x\left(t_{k}, \delta\right)=\hat{x}^{\delta}\left(t_{k}\right)$ of the base trajectory $x^{*}(\cdot)$ satisfy the relations

$$
\left\|\hat{x}^{\delta}\left(t_{k}\right)-x^{*}\left(t_{k}\right)\right\| \leq \delta
$$

$t_{0}=0 ; t_{k}=k \Delta t ; \quad k=1,2, \ldots, N ; \quad t_{N}=T$.

We put

$$
\delta \in\left(0, \delta^{*}\right], \quad \Delta t \in\left(0, \Delta^{*}\right] .
$$

We choose a constant $K_{1}>0$ and construct step-by-step, for instants $t_{k}, k=$ $1, \ldots, N$, continuous interpolations $y^{\delta}(t):[0, T] \rightarrow R^{n}$ of measurements $\hat{y}^{\delta}\left(t_{k}\right)$, $k=1, \ldots, N$, which are twice differentiable functions on each subinterval $\left[t_{k-1}, t_{k}\right]$, and satisfy the estimations:

$$
\left\|y^{\delta}(t)\right\| \leq K_{1}, \quad\left\|\frac{d y^{\delta}(t)}{d t}\right\| \leq K_{1} \quad \forall t \in[0, T]
$$




$$
\left\|\frac{d^{2} y^{\delta}(t)}{d t^{2}}\right\| \leq K_{1}, \quad \forall t \in[0, T] \backslash \Theta^{\delta}
$$

where the sets $\Theta^{\delta}$ have measures $\beta^{\delta}=\beta\left(\Theta^{\delta}\right)$, and $\beta^{\delta} \rightarrow 0$, as $\delta \rightarrow 0$. and the following relations are true:

$$
\left\|y^{\delta}(t)-x^{*}(t)\right\| \leq 2 \delta, \quad t \in[0, T] .
$$

Note, that we can do it as apply splines with nodes defined by $y^{\delta}\left(t_{k}\right)$.

The problem is: to reconstruct in real time a control generated the basic trajectory $x^{*}(\cdot)$ by the use of interpolations $y^{\delta}(\cdot)$ of incorrect measurements $\hat{x}^{\delta}\left(t_{k}\right)$.

\subsection{Hypothesis}

We consider the control reconstruction problem (1)-(3) under the following assumptions.

H1. Coordinates $g_{i j}(t, x), i \in \overline{1, n}, j \in \overline{1, m}$, of the matrix-function $G(t, x)$ are defined in the strip $\Pi_{T}=[0, T] \times R^{n}$, and there are no oscillating components of $g_{i j}(t, x)$; the functions $g_{i j}(t, x), \quad i \in \overline{1, n}, j \in \overline{1, m}$, are continuously differentiable in the domain $(0, T) \times R^{n}$, and their partial derivatives $\frac{\partial g_{i j}(t, x)}{\partial t}, \frac{\partial g_{i j}(t, x)}{\partial x_{k}}, k \in \overline{1, n}$ are extendable on any compact set $D \subset \Pi_{T}=[0, T] \times R^{n}$.

H2. There exist: a positive number $d_{0}$, and such a compact set $D_{0} \subset \Pi_{T}$ containing the graph of the basic trajectory $x^{*}(\cdot)$ that

$$
\left\{[0, T] \times\left\{x \in R^{n}:\|x\| \leq d_{0}\right\}\right\} \bigcap D_{0}=\emptyset,
$$

and the rank of the $m \times m$-matrix $\left\{g_{i, j}(t, x), i, j \in \overline{1, m}\right\}$ is equal to $m$ for all $(t, x) \in D_{0}$.

H3 For any $\Delta t \in(0, \Delta]$, there exist such numbers $\delta_{0} \in\left(0, \delta^{*}\right]$, and $\alpha^{*}>0$ satisfied the relations

$$
2 \delta_{0} \leq K_{1}(\Delta t)^{2}, \quad 4 \delta_{0}+\alpha^{*}<d_{0},
$$

where $K_{1}>0$ is defined by the set $D_{0}$ and assumptions H.1-H.2., that

$$
\Omega_{\delta}=\left\{(t, x):\left\|x-y^{\delta}(t)\right\| \leq 4 \delta+\alpha^{*}, t \in\left[t_{0}, T\right]\right\} \subset D_{0}, \quad \forall \delta \in\left(0, \delta_{0}\right] .
$$

\subsection{Statement of Control Reconstruction Problem}

Fix parameters $\Delta t \in\left(0, \Delta^{*}\right], \delta \in\left(0, \delta_{0}\right]$ and the an interpolation $y^{\delta}(\cdot)$ of current discrete incorrect measurements,

We consider the following Control Reconstruction Problem (CRP).

Problem 1. Find an admissible control $u^{\delta}(\cdot)$ generating the trajectory $x^{\delta}(\cdot)$ of system (1) step-by-step on the intervals $\left[t_{k-2}, t_{k}\right], k=2, \ldots, N$, to satisfy, at the end, the conditions:

- $C 1$.

$$
\left(t, x^{\delta}(t)\right) \in D_{0}, \quad \forall t \in[0, T]
$$

- $\quad C 2$.

$$
\lim _{\delta \rightarrow 0, \Delta t \rightarrow 0}\left\|x^{\delta}(\cdot)-x^{*}(\cdot)\right\|_{C}=0
$$

- $C 3$.

$$
\lim _{\delta \rightarrow 0, \Delta t \rightarrow 0}\left\|u^{\delta}(\cdot)-u^{*}(\cdot)\right\|_{L_{2}}=0 .
$$


Here the symbols $\|x(\cdot)\|_{C}$ and $\|u(\cdot)\|_{L_{2}}$ means the norms in the spaces $C\left([0, T], R^{n}\right)$ and $L_{2}\left([0, T], R^{m}\right)$. The "normal" control $u^{*}(\cdot)$ has the minimal norm in $L_{2}$ among all admissible controls generating $x^{*}(\cdot)$. Note, that the control reconstruction problem (1-(3) has the unique "normal" control under assumptions $H 1--H 3$.

\section{Auxiliary Constructions}

We suggest to use for solutions of CRP the following auxiliary variational problems.

\subsection{Calculus of Variations Problems}

We introduce a modified dynamical system of the form

$$
\frac{d x(\tau)}{d \tau}=\hat{G}(\tau, x(\tau)) v(\tau), \quad \tau \in\left[t_{k-2}, t_{k}\right]
$$

where controls $v=\left(u_{1}, \ldots, u_{m}, v_{m+1}, \ldots, v_{n}\right) \in R^{n}$ and $n \times n$ - matrix $\hat{G}(t, x)$ is constructed as

$$
\hat{g}_{i, j}(\tau, x)= \begin{cases}g_{i, j}(\tau, x), \quad i \in \overline{1, n}, \quad j \in \overline{1, m}, \\ 0, \quad i \in \overline{1, m}, \quad j \in \overline{m+1, n}, \\ 0, \quad i \in \overline{m+1, n}, \quad j \in \overline{m+1, n}, \quad i \neq j \\ \varepsilon, \quad i \in \overline{m+1, n}, \quad j \in \overline{m+1, n}, \quad i=j .\end{cases}
$$

where $\varepsilon$ is a small positive parameter of approximation.

Fix parameters $\Delta t \in(0, \Delta], \delta \in\left(0, \delta_{0}\right], \alpha>0, \varepsilon>0$. For any current instant $t_{k}, k \geq 2$, let us define an interpolation $y^{\delta}(t), t \in\left[t_{k-2}, t_{k}\right]$ of measurements and introduce the following cost discrepancy functional

$$
I^{\delta, \alpha}(x(\cdot), u(\cdot))=\int_{t_{k-2}}^{t_{k}}\left[\frac{\left\|x(t)-y^{\delta}(t)\right\|^{2}}{2}-\frac{\alpha^{2}\|v(t)\|^{2}}{2}\right] d t
$$

on the set $F$ of pairs of continuously differentiable functions $\left\{x(\cdot):\left[t_{k-2}, t_{k}\right] \rightarrow\right.$ $\left.R^{n}, u(\cdot):\left[t_{k-2}, t_{k}\right] \rightarrow R^{n}\right\}$. Here $\alpha>0$ is a small regularising parameter.

We consider pairs of continuously differentiable functions $\left(x(\cdot):\left[t_{k-2}, t_{k}\right] \rightarrow R^{n}\right.$, $\left.v(\cdot):\left[t_{k-2}, t_{k}\right] \rightarrow R^{n}\right) \ni F$ that satisfy differential equations (13) and the following terminal conditions

$$
x\left(t_{k-2}\right)=y^{\delta}\left(t_{k-2}\right), \quad \dot{x}\left(t_{k-2}\right)=\dot{y}^{\delta}\left(t_{k-2}\right) .
$$

We consider the following auxiliary Calculus of Variations Problem (CVP).

Problem 2. Find a pair of functions $\left\{x^{\delta, \alpha}(\cdot), v^{\delta, \alpha}(\cdot)\right\} \in F$ satisfied (13), (16) and provided a local minimum for the cost discrepancy functional (16). 


\subsection{CVP Necessary Optimality Conditions}

Necessary optimality conditions in CVP (13),(15),(16) provide the following Hamiltonian system:

$$
\begin{aligned}
& \dot{x}(t)=-\left(1 / \alpha^{2}\right) \hat{G}(x(t), t) \hat{G}^{T}(x(t), t) s(t), \\
& \dot{s}_{i}(t)=x_{i}(t)-y_{i}^{\delta}(t)+ \\
& +\left(1 / \alpha^{2}\right) s^{T}(t) \frac{\partial \hat{G}}{\partial x_{i}}(x(t), t) \hat{G}^{T}(x(t), t) s(t), \quad i \in \overline{1, n},
\end{aligned}
$$

where the vector $s(t)$ is the adjoint variables vector, the symbol ${ }^{T}$ denotes transposition, and the boundary conditions are satisfied

$$
x\left(t_{k-2}\right)=y^{\delta}\left(t_{k-2}\right), \quad \dot{x}\left(t_{k-2}\right)=\dot{y}^{\delta}\left(t_{k-2}\right) .
$$

\section{CRP Solutions}

In this section we describe an algorithm of construction of CRP solution. It is a modification of an algorithm described and justified in (Subbotina, 2019).

\subsection{Guiding Dynamics}

Let us consider on the interval $\left[t_{k-2}, t_{k}\right]$ the following linearized system obtained from (17)

$$
\begin{aligned}
& \dot{\bar{z}}(t)=-\left(1 / \alpha^{2}\right) Q_{k} \bar{s}\left(t_{k-2}\right)-\dot{y}^{\delta}\left(t_{k-2}\right), \\
& \dot{\bar{s}}(t)=\bar{z}(t),
\end{aligned}
$$

where $\bar{z}(t)=\bar{x}(t)-y_{i}^{\delta}(t)$,

$$
Q_{k}=\hat{G}\left(\bar{x}\left(t_{k-2}\right), t_{k-2}\right) \hat{G}^{T}\left(\bar{x}\left(t_{k-2}\right), t_{k-2}\right)
$$

and the following boundary conditions followed from (18)

$$
\bar{z}\left(t_{k-2}\right)=0, \quad \bar{s}\left(t_{k-2}\right)=\bar{s}^{k}\left(t_{k-2}\right)=\bar{s}^{k-1}\left(t_{k-2}\right)
$$

where the upper index $k$ means that the corresponding variable is obtained for the CVP on interval $\left[t_{k-2}, t_{k}\right], k=2, \ldots, N$, and

$$
\bar{s}\left(t_{0}\right)=-\alpha^{2} Q_{0}^{-1} \dot{y}^{\delta}\left(t_{0}\right)
$$

where $Q_{0}^{-1}$ is the inverse matrix for the non degenerate matrix

$$
Q_{0}=\hat{G}\left(y^{\delta}\left(t_{0}\right), t_{0}\right) \hat{G}^{T}\left(y^{\delta}\left(t_{0}\right), t_{0}\right) .
$$

\subsection{Guiding Control}

In problems (19)-(21), for fixed $\alpha, \delta$, we have obtained the guiding control

$$
v^{\delta, \alpha}(t)=-\left(1 / \alpha^{2}\right) \hat{G}^{T}\left(\bar{x}^{\delta, \alpha}\left(t_{k-2}\right), t_{k-2}\right) \bar{s}^{\delta, \alpha}(t),
$$

where $\bar{z}^{\delta, \alpha}(t), \bar{s}^{\delta, \alpha}(t)$, are solutions of system (19)-(21), $t \in\left[t_{k-2}, t_{k-1}\right), \bar{x}^{\delta, \alpha}\left(t_{k-2}\right)=$ $\bar{z}^{\delta, \alpha}\left(t_{k-2}\right)+y^{\delta}\left(t_{k-2}\right), \quad k=2, \ldots, N$, 
Let us introduce the cut-off function

$$
\hat{u}^{\delta, \alpha}(t)=\left\{\begin{array}{l}
{[v]_{m}^{\delta, \alpha}(t), \quad \text { as } \quad[v]_{m}^{\delta, \alpha}(t) \in U ;} \\
\hat{u} \in \arg \min _{u \in U}\left\|[v]_{m}^{\delta, \alpha}(t)-u\right\|, \quad \text { as } \quad[v]_{m}^{\delta, \alpha}(t) \notin U,
\end{array}\right.
$$

where $[v]_{m}=\left\{v_{1}, \ldots, v_{m}\right\}$ for $v \in R^{n}, n \geq m$.

We choose the functions $\hat{u}_{i}^{\delta, \alpha}(t), i \in \overline{1, m}, t \in\left[t_{k-2}, t_{k-1}\right], k=2, \ldots, N$ to consider them as solutions of inverse Prob.1.

\subsection{On Justifications}

This algorithm of solving Prob.1 is a modification and a simplification of an algorithm of solving CRP suggested and justified in (Subbotina, 2019). Namely, the new algorithm uses boundary conditions (18) for auxiliary dynamics (17). So, we need not construct inverse matrices $Q_{k}^{-1}$ for each subinterval $\left[t_{k-2}, t_{k}\right]$. Thiese procedure is made only one times (21) at the initial instant $t_{0}$. The novelity provides also better convergence of the method. Taking it in to account, one can modify schemes of proofs from (Subbotina, 2019) to get the following assertions.

Proposition 1. There exist such parameters $\delta^{0} \in\left(0, \delta_{*}\right], \varepsilon \in\left(0, \varepsilon_{0}\right], \alpha^{0}=\alpha\left(\delta^{0}, \Delta_{0}\right)$ that for all $\Delta t \leq \Delta_{0}, \delta \leq \delta^{0}, \varepsilon \leq \varepsilon^{0}, \alpha \leq \alpha^{0}$, solutions of system $\left(1 x^{\delta, \alpha}(\cdot)\right.$, started at the state $x^{\delta, \alpha}\left(t_{0}\right)=y^{\delta}\left(t_{0}\right)$ and generated by the control $\hat{u}^{\delta, \alpha}(t)$ (22), (22), are extendable on $[0, T]$ and $x^{\delta, \alpha}(t) \in D_{0}$.

Proposition 2. There exists the concordance of parameters $\Delta t, \delta, \alpha, \varepsilon$, such that the cut-off function $\hat{u}^{\delta, \alpha}(\cdot)$ of the form (22), satisfies conditions $C 1--C 3$, when $\Delta t \rightarrow 0, \delta \rightarrow 0, \alpha \rightarrow 0, \varepsilon \rightarrow 0$.

Note, that the last coordinates $v_{i}^{\delta, \alpha}(t), \quad i \in \overline{m+1, N}, t \in\left[t_{k-2}, t_{k-1}\right], k=$ $2, \ldots, N$ in (22) satisfy the relations

$$
\left\|v_{i}^{\delta, \alpha}(\cdot)\right\|_{C} \rightarrow 0, \quad i \in \overline{m+1, N}
$$

as $\Delta t \rightarrow 0, \delta \rightarrow 0, \alpha \rightarrow 0, \varepsilon \rightarrow 0$.

\section{Examples}

The following two examples illustrate simulation of solution of inverse problems in macroeconomics and navigation by the use of the new algorithm.

\subsection{Example 1. Control Reconstructions in Macroeconomics}

We consider a model of macroeconomic processes with dynamics

$$
\begin{aligned}
& \frac{d x_{1}(t)}{d t}=\frac{\partial G\left(x_{1}(t), x_{2}(t)\right)}{\partial x_{1}} u_{1}(t), \\
& \frac{d x_{2}(t)}{d t}=\frac{\partial G\left(x_{1}(t), x_{2}(t)\right)}{\partial x_{2}} u_{2}(t),
\end{aligned}
$$

$t \in[0, T], T=15, x_{1}(\cdot)$ is the production, $x_{2}(\cdot)$ is the production cost, $u_{1}, u_{2}$ are controls, and $G\left(x_{1}, x_{2}\right)$ is the profit,

$$
G\left(x_{1}, x_{2}\right)=x_{1} x_{2}\left(a_{0}+a_{1} x_{1}+a_{2} x_{2}\right) .
$$


Controlling parameters $u_{1}, u_{2}$ are restricted

$$
u_{1}^{2}+u_{2}^{2} \leq U
$$

where $U$ is a positive constant. The rate of taxation, the refunding rate and the currency exchange course are included in controlling parameters because they determine economical conditions for production activity in Russia. This model was suggested by E.H. Albrecht ${ }^{1}$

We use real statistic data on the work of industry of the Ural Region for the period 1970-1984 (10000 Rubles = 1).

Table 1. The Ural region's industry during 1970-1985.

\begin{tabular}{lll}
\hline Year & Gross Regional Product $x_{1, i}$ & Costs $x_{2, i}$ \\
\hline 1970 & 37.88 & 21.69 \\
1971 & 40.63 & 23.70 \\
1972 & 43.25 & 25.45 \\
1973 & 46.00 & 27.30 \\
1974 & 49.33 & 29.44 \\
1975 & 53.04 & 32.16 \\
1976 & 57.03 & 35.01 \\
1977 & 59.85 & 36.92 \\
1978 & 62.72 & 38.69 \\
1979 & 63.45 & 38.76 \\
1980 & 65.74 & 39.96 \\
1981 & 65.90 & 39.75 \\
1982 & 69.22 & 41.31 \\
1983 & 64.52 & 37.86 \\
1984 & 71.03 & 42.04 \\
1985 & 74.69 & 45.05 \\
\hline
\end{tabular}

We consider continuous piece-wise linear interpolation of the data as the basic trajectory $x_{1}^{*}(\cdot), x_{2}^{*}(\cdot)$, whose discrete measurements on interval $[0,15]$ become known in real time at instants $t_{k}$ with a fixed time step $\Delta t<1$. A spline interpolations $y_{1}^{\delta}(\cdot), y_{2}^{\delta}(\cdot)$ of the measurements is constructed.

The considering inverse problem is:

Problem 3. Reconstruct the controls $u_{1}^{*}(\cdot), u_{2}^{*}(\cdot)$, generating the basic trajectory $\left(x_{1}^{*}(\cdot), x_{2}^{*}(\cdot)\right)$, by known current measurements $y_{1}^{\delta}(\cdot), y_{2}^{\delta}(\cdot)$.

We apply the described above algorithm and get the following results.

We denote by the line $(a)$ the interpolation $y_{1}^{\delta}(\cdot)$, the line $(b)$ means the reconstructed trajectory $x_{1}^{\alpha, \delta}(\cdot)$, the line $(c)$ denotes the basic trajectory $x_{1}^{*}(\cdot)$ in pictures below. The similar results we get for variables $x_{2}$ and $u_{2}$. Here $T=15$.

\subsection{Example 2. A Flight at the Prescribed Altitude}

We consider the following model of a flight at the prescribed altitude from the book (Letov, 1969).

\footnotetext{
${ }^{1}$ Al'brekht, E.G. Methods of construction and identification of mathematical models for macroeconomic processes.// Electronic Journal "Investigated in Russia", http://zhurnal.ape.relarn.ru/articles/2002/005.pdf (in Russian)
} 


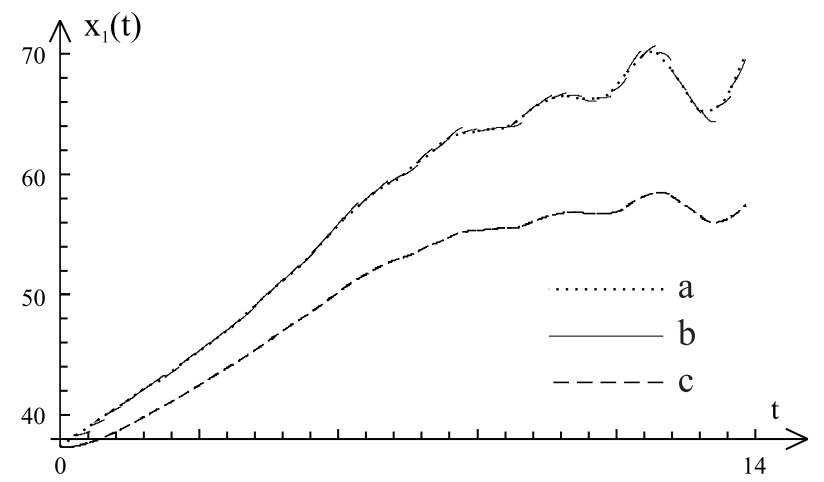

Fig. 1. reconstructed trajectory $x_{1}^{\alpha, \delta}(\cdot), \delta=0.1, \alpha=0.1, \Delta t=T / 50$.

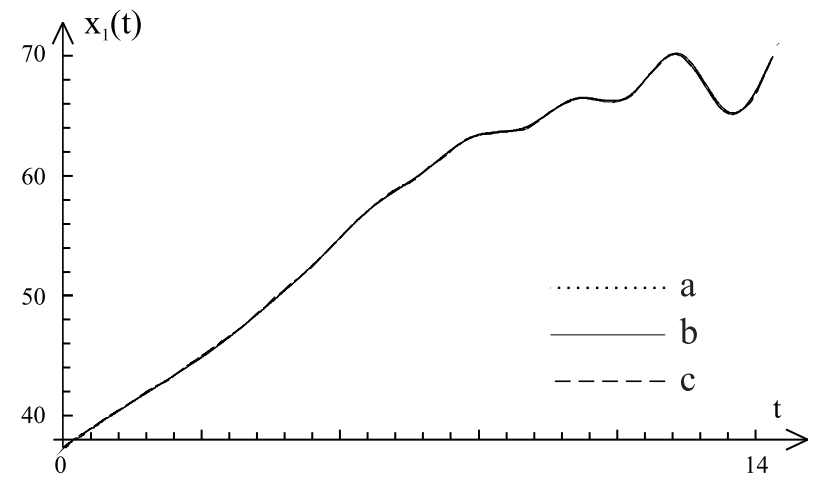

Fig. 2. reconstructed trajectory $x_{1}^{\alpha, \delta}(\cdot), \delta=0.1, \alpha=0.1, \triangle t=T / 100$.

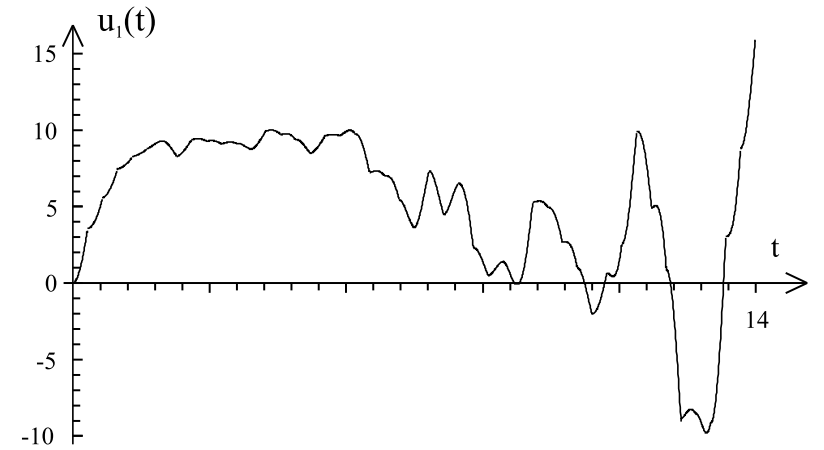

Fig. 3. reconstructed control $\hat{u}_{1}^{\delta, \alpha}(\cdot), \delta=0.1, \alpha=0.1, \Delta t=T / 50$. 


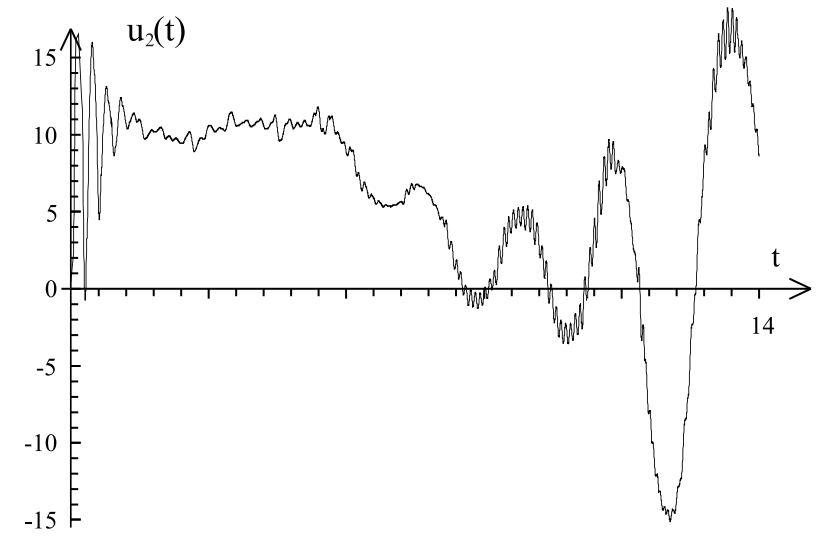

Fig. 4. reconstructed control $\hat{u}_{1}^{\delta, \alpha}(\cdot), \delta=0.01, \alpha=0.01, \Delta t=T / 100$.

The dynamics of flight is:

$$
\begin{aligned}
\frac{d x_{1}}{d t} & =x_{2} \\
\frac{d x_{2}}{d t} & =\frac{c u-Q\left(x_{2}\right)}{m(t)}
\end{aligned}
$$

Here $x_{1}$ is the great-circle arc starting from a certain point, $x_{2}$ is the ship velocity along its trajectory, $m(t)$ is fuel mass, $m(t) \geq m_{0}>0$, control $u$ is the fuel consumption. The following restrictions are satisfied:

$$
u \in U=\{0 \leq u \leq \beta\},
$$

The symbol $Q$ denotes the aerodynamic drag defining via the formula (Letov, 1969)

$$
Q=c_{x} p S \frac{\left(x_{2}\right)^{2}}{2}
$$

the symbol $S$ denotes the wing area, $p$ is the the air density, known as a function of altitude $H$ at a constant temperature, the symbol $c_{x}$ means the drag coefficient, which is given by the known function of

$$
M=\frac{x_{2}}{a},
$$

where $a$ is equal to the sound speed.

We take into consideration the basic trajectory $\left(x_{1}^{*}(t), x_{2}^{*}(t)\right)$ simulated by the use of the following control

$$
u^{*}(t)=\left\{\begin{array}{l}
10,0 \leq t<2.5 \\
10+10 \sin (t), 2.5 \leq t \leq 10 .
\end{array}\right.
$$

Watching the flight $\left(x_{1}^{*}(t), x_{2}^{*}(t)\right)$, we get in real time inaccurate discrete state measurements $\left(x_{1}\left(t_{j}\right), x_{2}\left(t_{j}\right)\right)$ :

$$
\left\|x_{i}\left(t_{j}\right)-x_{i}^{*}\left(t_{j}\right)\right\| \leq \delta, \quad i=1,2,
$$


$t_{0}=0<t_{1}<t_{2}, \ldots,<t_{N}=T, \delta>0$.

Continuous interpolations $\left(y_{1}^{\delta}(t), y_{2}^{\delta}(t)\right)$ of the measurements are constructed as splines.

The above presented method is applied to obtain the reconstruction $u^{\alpha, \delta}(t)$ of the control $u^{*}(t)$ with a small delay in time $\Delta t$ in real time.

We put $S=10, p=0.526\left(\mathrm{~kg} / \mathrm{m}^{3}\right), H=8(\mathrm{~km}), \quad M=0.4, c_{x}=0.85$.

Results of simulations of solution of the control reconstruction problem are exposed at the pictures below.

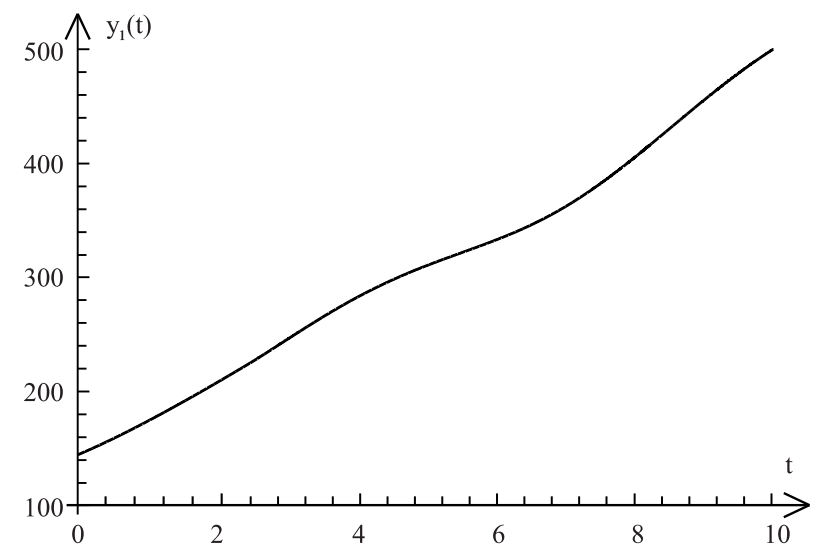

Fig. 5. interpolation of measurements $y_{1}^{\delta}(\cdot)$

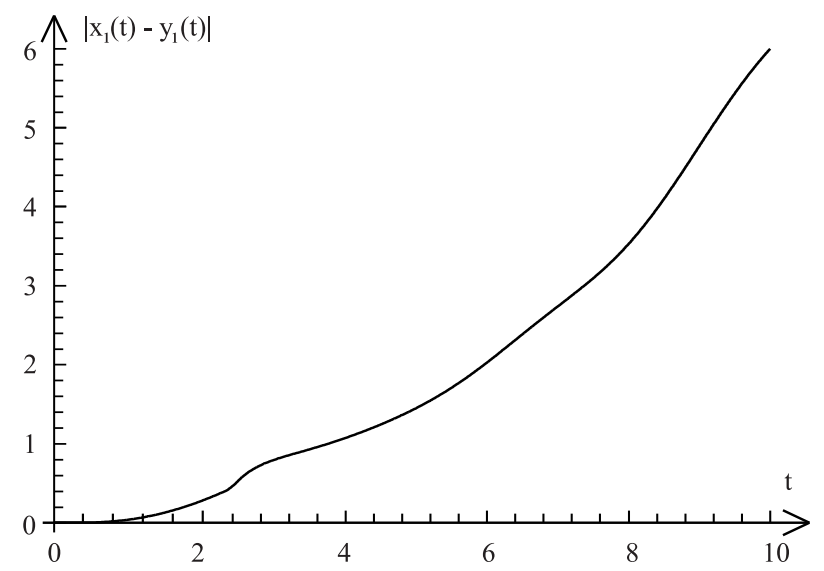

Fig. 6. difference between interpolation $y_{1}^{\delta}(\cdot)$ and reconstructed trajectory $x_{1}^{\alpha, \delta}(\cdot)$ 


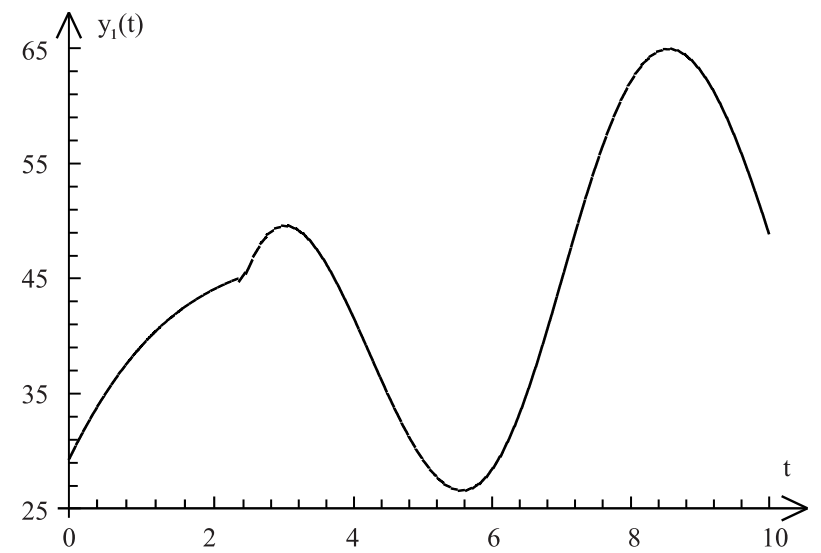

Fig. 7. interpolation of measurements $y_{2}^{\delta}(\cdot)$

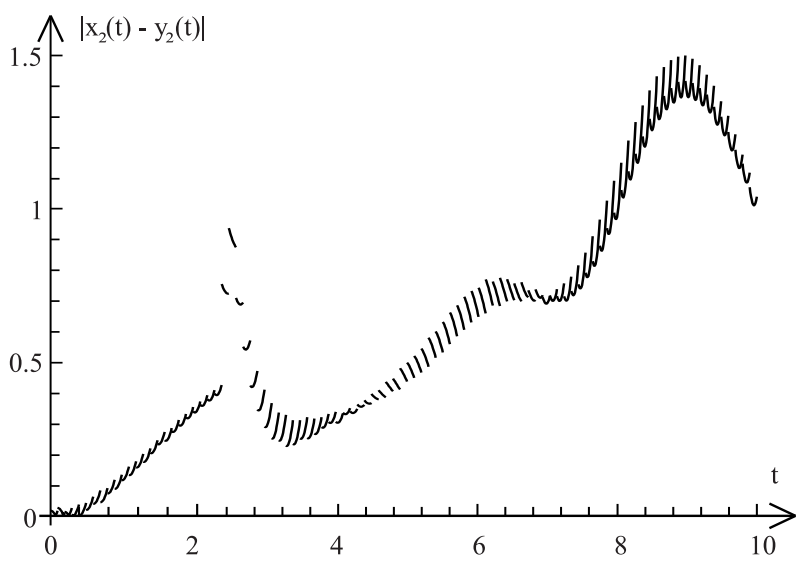

Fig. 8. difference between interpolation $y_{2}^{\delta}(\cdot)$ and reconstructed trajectory $x_{2}^{\alpha, \delta}(\cdot)$

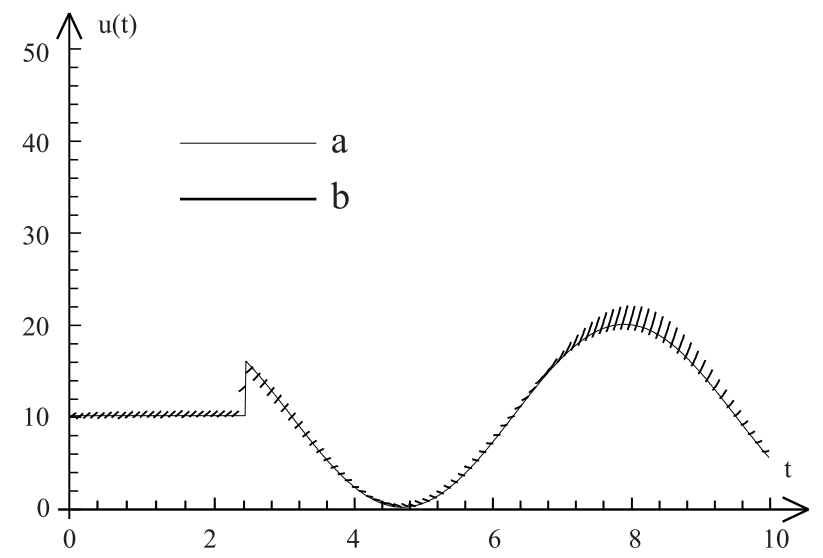

Fig. 9. unknown control $u^{*}(\cdot)\left(\left(\right.\right.$ a) line) and reconstructed control $u^{\alpha \delta}(\cdot)((\mathrm{b})$ line) 


\section{Conclusion}

In the paper, the new method is presented to solve dynamic reconstruction problems in real time using information about inaccurate current state measurements. This method relies on necessary optimality conditions in auxiliary variational problems with concave-convex integral discrepancy functional. A new algorithm of numerical solution of inverse problems is suggested. Results of simulation of solution of inverse problems in macroeconomics and navigation are exposed.

Let us underline, that realization of the algorithm requires standard mathematical tools and software. The new feature of it is that the inverse matrix $Q_{0}^{-1}$ is calculated only at the beginning of the reconstruction process. One needn't calculate matrixes $Q_{k}^{-1}$ on each step of the reconstruction process. This method has also discovered properties of stability relative to perturbations to input data. All of it makes the suggested procedure great effective.

Acknowlegments. The author express her gratitude to Eugenii A. Krupennikov and Timofey B. Tokmantsev for simulation and graphic presentation of results for the illustrative examples above.

\section{References}

Bellman, R. (1957). Dynamic Programming. Princeton University Press: Princeton, NJ.

D'Autilia, M. C., Sgura, I., and B. Bozzini (2017). Parameter identification in ODE models with oscillatory dynamics: a Fourier regularization approach. Inverse Problems, 33(6), 124009, IOP Publishing Ltd.: NY.

Krasovskii, N. N. (1968). The Theory of Motion Control. Nauka: Moscow (in Russian).

Krasovskii, N.N. and A.I. Subbotin (1988). Game-Theoretical Control Problems., Springer-Verlag: NY.

Krupennikov, E. A. (2018). On Control Reconstruction Problems for Dynamic Systems Linear in Controls. In: Frontiers of Dynamic Games. Game Theory and Management (Petrosyan, L. A., Mazalov, V. V. and N. A. Zenkevich. eds.), pp. 89-120. Birkhäuser: Boston.

Kryazhimskii, A. V. and Yu.S. Osipov (1984). Modelling of a control in a dynamic system. Eng. Cybern., 21(2), 38-47.

Leitmann, G. (1962). In: Optimization techniques: with applications to aerospace systems (Leitmann, G. ed.), Vol. 5. Academic Press: NY.

Letov. A. M. (1969). Dynamics of Flight and Control Nauka: Moscow (in Russian).

Liu, Y.C., Chen, Y.W., Wang, Y.T. and Chang, J.R. (2018). A high-order Lie groups scheme for solving the recovery of external force in nonlinear system. Inverse Problems in Science and Engineering, 26(12), 1749-1783, DOI: 10.1080/17415977.2018.1433669.

Michel, A. (1977). Deterministic and stochastic optimal control. IEEE Trans. Automat. Contr., 22(6), 997-998.

Osipov, Y.S. and A. V. Kryazhimskii (1995). Inverse Problems for Ordinary Differential Equations: Dynamical Solutions Gordon and Breach: Amsterdam.

Osipov, Yu.S., Kryazhimskii, A. V. and V. I. Maksimov (2011). Some Algorithms for the Dynamic Reconstruction of Inputs. Proc. Steklov Inst. Math. 275(Suppl.1), 86-120.

Pontryagin, L. S. Boltyansky, V. G. Gamkrelidze, R. V. and E. F. Mishchenko (1961). The Mathematical Theory of Optimal Processes. Nauka: Moscow (in Russian).

Petrosjan, L. A. and N.A. Zenkevich (1996). Game Theory. World Scientific Publisher: NY.

Sabatier, P.C. (2000). Past and future of inverse problems. J. Math. Phys., 41, 4082, DOI:10.1063/1.533336. 
Subbotina, N. N. (2019). On solutions of navigation inverse problems in real time. AIP Conference Proceedings, 020019, DOI (CrossRef): 10.1063/1.5135679. AIP Publishing: NY.

Subbotina, N. N. and E. A. Krupennikov (2017). The method of characteristics in an identification problem. Proc, Steklov Inst. Math., 299(Suppl. 1), 205-216.

Subbotina, N. N. and E. A. Krupennikov (2018). A Solution to Inverse Problems For Dynamic Control Systems. AIP J. Proceed., 1997(1), 020006.

Subbotina, N. N. and T. B. Tokmantsev (2013). The Method of Characteristics in Inverse Problems of Dynamics. Universal J. Contr. Autom., 1(3), 79-85.

Subbotina, N. N., Tokmantsev, T. B. and E. A. Krupennikov, (2015). On the solution of inverse problems of dynamics of linearly controlled systems by the negative discrepancy method. Proc. Steklov Inst. Math., 291, 253-262.

Tikhonov, A. N. (1963). Solution of incorrectly formulated problems and the regularization method. Sov. Math., Dok., 4, 1624-1627. 